\title{
ORIGEN Y PERSPECTIVAS DE LA ATENCION PRIMARIA EN SALUD
}

\author{
José Rubén Castillo Garcia
}

T. S. y Mgr. en «Desarrollo Educativo y Socia . Profesor Asociado Facultad de Fisioterapia. U. A. M.

1 tema de la «Atención Primaria en Salud» ha cobrado gran actualidad, sin embargo, se ha vuelto tan obvio, que el espíritu de sus contenidos, en muchos casos se ven distanciados de la pretensión inicial de sus impulsores, y en ciertas ocasiones se manejan con los mismos términos y perspectivas contrarias desde el punto de vista del propósito que le dió origen. Por esto, parece importante hacer un análisis sobre las condiciones y circunstancias que condujeron a los representantes de algunos Estados y de ciertas instituciones encargadas de velar por la salud de la población para que generaran, definieran e impulsaran esta estrategia.

\section{ANTECEDENTES}

Ubiquémonos en los comienzos de la década de los años setenta $(1970 \ldots)$.

En esta época, se hacía notoria la desigualdad social, originada en la inadecuada redistribución de la riqueza. 
$\mathrm{Al}$ parecer los modelos de desarrollo que se apoyaron en el crecimiemto económico y en la industrialización al margen de las necesidades de la población, tuvieron como resultado entre otros procesos, la emigración de los campesinos hacia las ciudades, buscando con ello resolver sus necesidades básicas. Pero aconteció que se magnificaron los problemas de subsistencia y se hizo mayor la brecha entre los protegidos por la fortuna y los marginados.

Los grupos más vulnerables, se hicieron más desprotegidos y se presentaron entre ellos diferentes formas de expresar la inconformidad, (huelgas, tomas de tierras, paros cívicos, movimientos guerrilleros, etc.), lo cual hizo de este período un momento crítico en el desarrollo de estas sociedades.

Por lo anterior, la Asamblea General de las Naciones Unidas (O. N. U.), en 1974, expresó la necesidad de velar por el mejoramiento de las condiciones de vida de la población, dado que se evidenciaba el incremento en las diferencias sociales, creando y consolidando el caldo de cultivo para la presencia de múltiples movimientos de insatisfacción popular.

La Organización Panamericana de la Salud O. P. S (oficina Regional de la Organización Mundial de la Salud O. M. S) expresó sin rodeos sus puntos de vista al respecto.Veamos:

«La pobreza es el problema de más importancia en los países de América Latina y el Caribe. La pobreza acarrea consigo graves problemas de salud. En 1972, aproximadamente el $40 \%$ de una población de 283 millones no tenía acceso a servicios para atender su salud.

...Los grupos migratorios procedentes de las zonas rurales de distintas características forman, de ordinario, los cinturones de miseria de las grandes ciudades. Allí se organizan para satisfacer sus necesidades inmediatas de vivienda y trabajo... Se calcula que en el año 2.000 , el $75 \%$ de la población vivirá en las ciudades.

... La pobreza, el hacinamiento en viviendas insalubres, la ignorancia sanitaria, el analfabetismo, el desempleo o subempleo y las creencias y costumbres populares, contribuyen a mantener ciertas prácticas y hábitos de la población que afectan seriamente la salud. (1)

Teniendo como base esta descripción sobre las condiciones en las 
cuales se desenvolvía la vida de la población y que caracterizaban su marginalidad, se hacía necesario establecer la situación que se presentaba en el campo de la salud, en aras de identificar las posibilidades, las dificultades y/o las limitaciones presentes en su funcionamiento.

Se critica la incapacidad de algunos gobiernos para darle solución a las necesidades de la población en esta área, la poca cobertura lograda con sus servicios, el elevado costo de los mismos, la multiplicidad y descoordinación de los esfuerzos institucionales; el marginamiento de la población en los programas destinados a proveerlos de vida sana. Se veía la necesidad de reorientar la manera de conducir los «Sistemas nacionales» encargados de atender el sector de la salud.

\section{SALUD PARA TODOS EN EL AÑO 2000}

Cuando la $30^{\circ}$ Asamblea Mundial de la Salud se reunió en mayo de 1977, estaban dadas las condiciones para que se asumieran decisiones urgentes que tendieran a buscar soluciones a dicha problemática, so pena de incrementar el caldo de cultivo para que se generaran y desarrollaran conflictos sociales de tendencia subversiva, que condujeran al deterioro de los Estados y de la estabilidad política de los países en esta región.

En este contexto, se define la META de «Salud para todos en el año 2000", asumida por los 152 Estados miembros de la O. M. S. También se expresa cierta claridad sobre su significado. Quedaba latente la necesidad de resolver la manera de intervenir para efectos de lograrla. Esa necesidad fue la de establecer una estrategia que permitiera avanzar en la eficacia y en la eficiencia de las acciones tendientes a la atención de la salud de la población en condiciones óptimas.

Por ello, se llevaron a cabo varios eventos preparatorios, diversas reuniones nacionales, regionales e intercontinentales, las cuales se efectuaron entre 1977 y 1978 y dieron origen a la estrategia de la Atención Primaria en Salud.

\section{LA ESTRATEGIA DE «LA ATENCION PRIMARIA EN SA- LUD»}

En las condiciones y circunstancias anteriores surge esta propuesta. Tiene la intención de darle solución efectiva a la problemática social y de salud de la población a la cual se ha hecho referencia. En términos de la O. M. 
S. y de la O. P. S. la propuesta de la Atención Primaria en Salud, se basa en tres elementos.

... El PRIMERO, la aceptación y la adecuación de las acciones que la comunidad tradicionalmente ejecuta para la atención de su salud.

... El SEGUNDO, la reorientación de las acciones del sistema institucional de salud. Se entiende como tal el conjunto de organismos públicos e instituciones o personas privadas.

... El TERCERO, el desarrollo de mecanismos de articulación entre el sistema institucional y el sistema tradicional comuntario, para asegurar el acceso permanente de la población a todos los niveles de atención del sistema institucional. (2)

Sin embargo, se hace claro y evidente que no basta con las buenas intenciones de las entidades de los Estados, encargadas tradicionalmente de prestar los servicios de salud a la población, dado que allí hay un papel fundamental que debe cumplir la población sujeto de las acciones de salud.

De acuerdo con el informe de un comité de expertos de la O. M. S. se considera que el SENTIDO BASICO de la Atención Primaria es:

... la asistencia sanitaria esencial basada en métodos y tecnologías prácticos, científicamente fundados y socialmente aceptables, puesto al alcance de todos los individuos y familias de la comunidad mediante su plena participación y a un costo que la comunidad y el país puedan soportar...

... La atención primaria forma parte intregante tanto del sistema nacional de salud, del que constituye la función central y el núcleo principal, como del desarrollo social y económico global de la comunidad. Representa el primer nivel de contacto de los individuos, la familia y la comunidad con el sistema nacional de salud, llevando lo más cerca

-...educación sobre los principales problemas de salud y sobre los métodos de prevención y lucha correspondientes; 9 
posible la atención de salud al lugar donde residen y trabajen las personas, y constituye el primer elemento de un proceso permanente de asistencia sanitaria. (3) y traumatismos comunes; y el suministro de medicamentos esenciales». (4)

El comité de expertos de la O. M. S. consideró que en la definición de la «Atención Primaria en Salud» se materializan entre
Es de recordar que el texto de la declaración presenta ocho elementos considerados fundamentales, para efectos de la aplicación y del desarrollo de la Estrategia:

«Comprende, cuando menos las siguientes actividades: La educación sobre los principales problemas de salud y sobre los métodos de prevención y lucha correspondientes; la promoción del suministro de alimentos y de una nutrición apropiada; un abastecimiento adecuado de agua potable y saneamiento básico; la asistencia maternoinfantil con inclusión de la planificación de la familia; la inmunización contra las principales enfermedades infecciosas; la prevención y la lucha contra las enfermedades endémicas locales; el tratamiento apropiado de las enfermedades otros los siguientes PRINCIPIOS:

- La distribución equitativa (Recur sos y posibilidades)

- La participación cie la comunidad

- La tecnología adecuada o apropiada

- la primacía de la prevención y la multi sectoriali dad (Cooperación)
El comité observó que en 1981 la $34^{\circ}$ Asamblea Mundial de la Salud adoptó la resolución WHA34.36 sobre una estrategia mundial de salud para todos en el año $2000 . .$. La estrategia está fundada en algunas POLITICAS definidas por la O. M. S.

- La defensa del derecho fundamental a la salud, concebido como un derecho humano y una meta social mundial. 


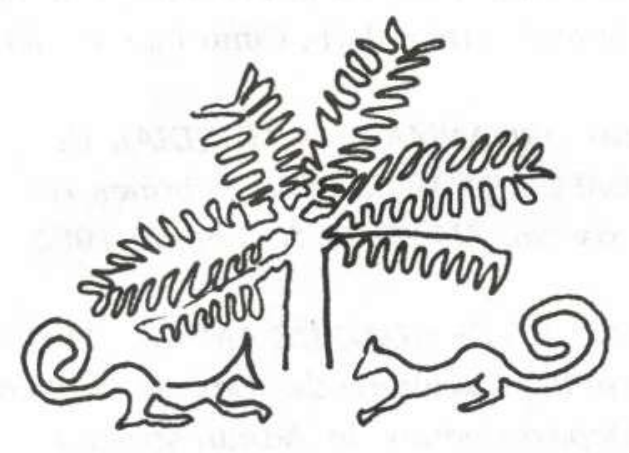

- La salud debe ser entendida como parte integrante del desarrollo.

- Una distribución equitativa de los recursos sanitarios.

- La participación de la comunidad, individual y colectivamente en la planificación y aplicación de la asistencia sanitaria.

- El compromiso político de la Nación en su conjunto, y no sólo del Ministerio de Salud.

- La autorresponsabilidad nacional, es decir, que los países deben llegar a ser independientes, aunque no necesariamente autosuficientes en cuestiones de salud.

- La cooperación técnica y económica entre los países y la coordinación entre los sectores para que pueda apoyar el desarrollo económico y social.

- Comprende medidas concretas que deben aplicar las personas y las familias en sus hogares y en las comunidades.

- Presupone elegir la tecnología adecuada para el país que se trate, en el sentido de que esté fundada científicamente, se pueda adaptar a las distintas circunstancias locales... y pueda mantenerse con los recursos de que disponga el país.

- Que logre el control social de la infraestructura y la tecnología de salud mediante la activa participación de la comunidad. (5)

Por lo anterior y teniendo en cuenta las circunstancias sociales en las cuales viven nuestras poblaciones en la actualidad, se considera que las condiciones en las cuales se generó la META de «Salud Para Todos en el año 2000», la propuesta y los contenidos de la ESTRATEGIA de "Atención Primaria en Salud», continuan vigentes y que es deber de los diferentes profesionales, funcionarios, instituciones y personas que tienen que ver con la solución de las necesidades de salud de la población, luchar para lograr la 
aplicación de los propósitos de esta propuesta.

Para ello, es necesario rescatar su Espíritu, sus Criterios, Enfoque. Metodologías, Principios, Políticas y con base en ésto, promover la implementación de dicha estrategia en el Sistema Sanitario de los países adscritos a la O. M. S.

(1) ORGANIZACION PANAMERICANA DE LA SALUD (O. P. S.) «La Participación de la Comunidad en la Prestación de los Servicios de Salud". Cinta Fija No. 73. División de Servicios de Salud de la O.P. S. 1977
(2) ORGANIZACION PANAMERICANA DE LA SALUD (O. P. S.) «La Participación de la Comunidad en la Prestación de los Servicios de Salud». Cinta Fija No. 73.

(3) ORGANIZACION MUNDIAL DE LA SALUD (O. M. S.) Serie «Informes Técnicos» No. 717. O. M. S. Ginebra 1985

(4) UNIVERSIDAD DE ANTIOQUIA. Escuela Nacional de Salud Pública. Departamento de Administración de Salud. «Atención Primaria en Salud». M2576 Numeral VII-3

(5) ORGANIZACION MUNDIAL DE LA SALUD. (O. M. S.). Serie «nformes Técnicos No. 708, Ginebra 1984. Pág. 10-12

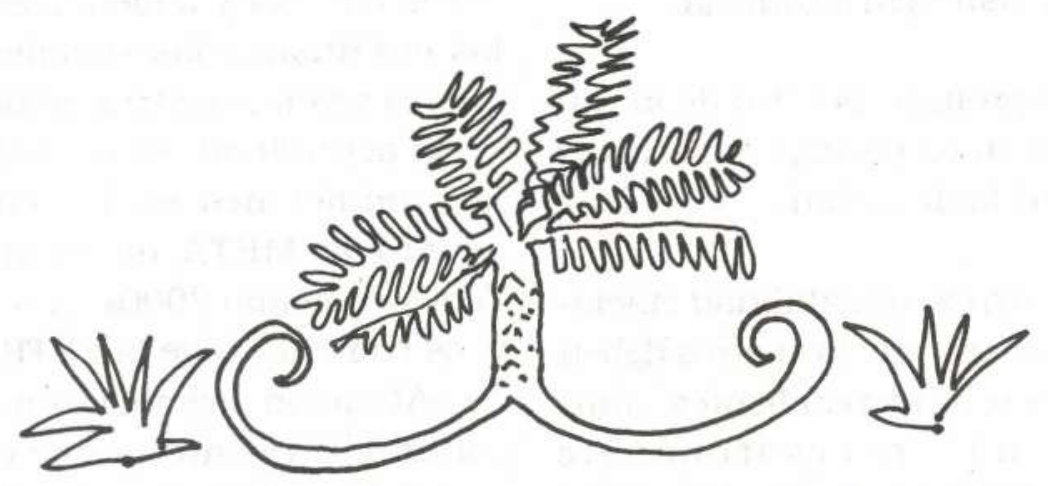

